\title{
Enhancing the Detector for Advanced Neutron Capture Experiments
}

\author{
A. Couture ${ }^{1, a}$, S. Mosby ${ }^{1}$, B. Baramsai ${ }^{1}$, T. A. Bredeweg ${ }^{1}$, M. Jandel ${ }^{1}$, K. Macon ${ }^{2}$, J. M. O’Donnell ${ }^{1}$, G. Rusev ${ }^{1}$, T. N \\ Taddeucci $^{1}$, J. L. Ullmann ${ }^{1}$, and C. L. Walker ${ }^{1}$ \\ ${ }^{1}$ Los Alamos National Laboratory, PO Box 1663, Los Alamos, NM 87545, USA \\ ${ }^{2}$ Department of Physics and Astronomy, Louisiana State University, 202 Nicholson Hall, Tower Dr., Baton Rouge, LA 70803, USA
}

\begin{abstract}
.
The Detector for Advanced Neutron Capture Experiments (DANCE) has been used for extensive studies of neutron capture, gamma decay, photon strength functions, and prompt and delayed fission-gamma emission. Despite these successes, the potential measurements have been limited by the data acquisition hardware. We report on a major upgrade of the DANCE data acquisition that simultaneously enables strait-forward coupling to auxiliary detectors, including high-resolution high-purity germanium detectors and neutron tagging array. The upgrade will enhance the time domain accessible for time-of-flight neutron measurements as well as improve the resolution in the DANCE barium fluoride crystals for photons.
\end{abstract}

\section{Introduction}

The Detector for Advanced Neutron Capture Experiments (DANCE) is a $4-\pi \mathrm{BaF}_{2}$ scintillator array that is optimized for performing neutron-capture experiments on small, radioactive samples [1]. DANCE is coupled to a $20 \mathrm{~m}$ flight-path station at the Manuel Luján Neutron Scattering Center at the Los Alamos Neutron Science Center (LANSCE) [2, 3]. This water-moderated flightpath provides approximately time-of-flight neutrons at a rate of $3 \times 10^{5} \mathrm{n} / \mathrm{s} / \mathrm{cm}^{2} /$ decade neutron energy over a $7 \mathrm{~mm}$ diameter beam spot at the DANCE sample position. DANCE has been successfully used for neutron-capture cross-section measurements with applications to nuclear astrophysics, nuclear energy, and defense programs on a range of stable and radioactive samples from thermal to several hundred $\mathrm{keV}$ incident neutron energy e.g. Refs. [4-7]. Recently, the experiments at DANCE have taken increased advantage of the calorimetric nature of the measurements in order to study additional physical properties of the atomic nucleus, including the photon strength function (PSF) as well as prompt gamma emission following neutroninduced and spontaneous fission e.g. Refs. [8-10].

All of this has been accomplished with a data acquisition system (DAQ) based on 320 channels of Acqiris (now Agilent) DC265 digitizers, which are 8-bit resolution, $500 \mathrm{MSample} / \mathrm{s}$ sampling frequency, $128 \mathrm{kSam}$ ple/channel memory digitizers built on a cPCI platform. They have no on-board waveform processing capability. At most 28 channels can share a common sampling clock. The digitizer memory limitations mean that segments of at most $256 \mu$ s of time-of-flight neutron information can be recorded. For reference, $25 \mathrm{meV}$ neutrons (thermal) arrive

\footnotetext{
a e-mail: acouture@lanl.gov
}

at approximately $9.2 \mathrm{~ms}$. Acquisition of this limited data is only possible with the use of a single-board computer for each 24 data channels. Seventy percent of the "processing" time between beam pulses is dedicated to transferring this small portion of the interesting data over the PCI bus so that the single board computer can process the extract the physics of interest and pass it on to the archiver. Details of the existing DAQ are available in Ref. [11].

\section{DAQ Upgrade}

DANCE is presently undergoing a major upgrade to the data acquisition system. This will offer several major improvements. In particular, we expect to improve the gamma-ray energy resolution, particularly for gamma rays of energies $E_{\gamma}<500 \mathrm{keV}$. We plan to take advantage of improvements in onboard field-programmable gate array (FPGA) waveform processing and memory to allow acquisition over the entire $50 \mathrm{~ms}$ between beam spills rather than the present limit of $\approx 500 \mu \mathrm{s}$. All of the present digitizers are being replaced with CAEN VX1730 digitizers. The VX1730 series digitizers have many advantages, including improved resolution, improved data transfer speeds, and a more standard nuclear physics platform, but the biggest single improvement will come from the onboard FPGA which will allow peak identification and data extraction on the digitizer board itself, which drastically lowers the demands on the data bus between the digitizer and the computer. The impact of this is illustrated graphically in Fig. 1, which shows the range of possible experiment that can be completed with the new boards compared to the old. Another significant advantage will come from the ability to run all digitizers on a single, common clock, reducing channel-to-channel jitter and greatly simplifying the 


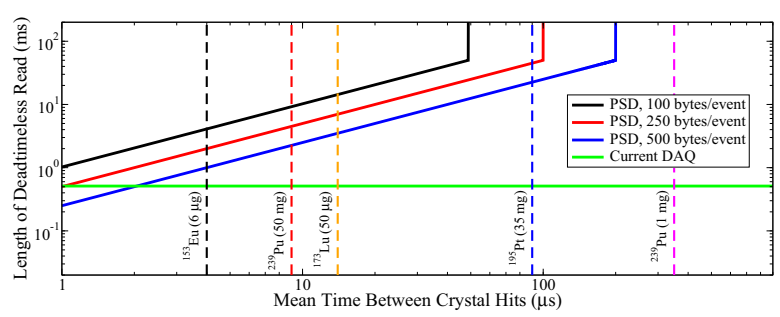

Figure 1. Shown above is a plot illustrating the improvements the upgrade will offer over the existing system. Shown in green is the present limit of $512 \mu \mathrm{s} / 50 \mathrm{~ms}$. The black, blue, and red solid lines indicate the total acquisition time as a function of event rate for different assumptions on how much waveform is saved by the PSD firmware on the FPGA. The vertical dashed lines indicate samples that have been run at DANCE in the past.

clock synchronization. Finally, the use of vendor-supplied firmware in the onboard will greatly simplify the addition of auxiliary detectors as discussed in Sec. 3.

The DAQ is being implemented in a MIDAS framework in order to take advantage of the flexibility of the MIDAS approach [12]. In the initial implementation, each of the 196 digitizer channels (16 channels on 12 boards) will be run as, effectively, an independent data acquisition system. Each channel will run with the standard pulseshape discrimination (PSD) firmware from CAEN to selftrigger on events in the crystals, determine an event time, extract peak information, store a portion of the waveform, and transfer a structure containing these data to the master computer. At this stage, there is no physics definition of an event aside from knowing that a particular detector number saw an event with certain properties at a given time. This event time, derived from the synchronized digitizer 64-bit timestamp, is the single, unifying datum that allows event reconstruction across the detector data streams.

Initial tests of the approach and algorithms have already been performed with earlier generation CAEN digitizers [13]. While the full algorithms used for DANCE analysis have not yet been developed, initial results have already shown that reconstruction of crystal multiplicity and total gamma energy are consistent with the old DANCE DAQ in gamma-ray source tests. In the fall and winter of 2014/early 2015 , both data acquisition systems will be run in parallel for most experiments in order to optimize beamtime and development of the DAQ.

\section{Auxiliary Detectors}

The addition of auxiliary detectors to the DANCE system opens the door both to expand cross section measurement campaigns as well as new physics that can be studied. Parallel-Plate Avalanche Counter (PPAC) detectors have been used with DANCE in the past as fission tagging detectors to separate the fission gamma component from the capture signal $[14,15]$. While this has been successful, the PPAC signal was shaped with analog electronics to conform to the filtering algorithms for the $\mathrm{BaF}_{2}$ signals, a crude, if effective, approach. To improve the performance of the PPAC, it would be desirable to customize the data acquisition to the signal, which is trivial with the new data acquisition. Further, plans are underway to instrument a small, liquid scintillator array inside of DANCE that will be used for high-energy ( $>500 \mathrm{keV}$ ) neutron detection as an alternative fission tag. This will require pulse shape discrimination. Existing algorithms for DANCE are neither flexible for adding the $\sim 20$ acquisitions channels nor offer the ability to customize integral windows for liquid scintillator. While none of these issues are fundamental barriers, the upgraded system will offer channel by channel customization of the filtering through the FPGA as well as simple mechanism to add additional detector data streams without the addition of data processing computers.

Finally, for some applications, it is very advantageous to replace a small number of $\mathrm{BaF}_{2}$ crystals with highresolution detectors, such as HPGe. Because the detector pulse shapes are so different, the waveform analysis algorithms are quite different. Further, even though sampling the full waveform can compensate for limitations in resolution, the 8-bit digitizer used before did not have the needed resolution to take advantage of the resolution offered by the HPGe detectors. The new digitizers, with 14-bit resolution are much better suited to high-resolution applications, even if the full 500 MSamples/s are not required. Further, pulse-height analysis firmware, which is presently available for the VX1730 in beta stage, is ideal for analyzing these signals.

One of the remaining challenges facing the integration of the HPGe detectors with DANCE comes from the need to perform a precise time reconstruction. Typical event reconstruction windows are on the order of $10 \mathrm{~ns}[6,7]$. Signals from $\mathrm{HPGe}$ are much slower than $\mathrm{BaF}_{2}$ signals and have pulse-shape defects that interfere with precise timing.

In order to test our ability to maintain reasonable time reconstruction together with $\mathrm{HPGe}$ detectors, we tested data acquisition of a hybrid $\mathrm{BaF}_{2}$ and $\mathrm{HPGe}$ array with a prototype CAEN N6730 digitizer with beta PSD firmware. The CAEN N6730 is the same base digitizer packaged on a NIM platform. Only eight channels are available, and the digital I/O for trigger decision are more limited than on the VX1730. However, the digitizer performance should be the same. In these tests, a single $\mathrm{BaF}_{2}$ crystal was replaced with a $35 \%$ coaxial HPGe detector. Neutron capture measurements were performed with either a ${ }^{195} \mathrm{Pt}$ or a ${ }^{239} \mathrm{Pu}$ sample in DANCE. Because only eight channels of DAQ were available on this prototype, full cascade reconstruction was not possible. However, it was possible to measure the coincidence time reconstruction and $\mathrm{HPGe}$ energy resolution.

For the prototype board and firmware tests, the PHA firmware was not available, so the PSD firmware was used. The HPGe preamp output was directly digitized. The firmware allowed us to capture 1000 samples, or $2 \mu$ s of waveform, for each event. An offline waveform fitting routine was developed which extracted both energy and time from the HPGe signal. There were not sufficient sample to achieve intrinsic HPGe resolution $(<2 \mathrm{keV})$, but resolutions of $\sim 4 \mathrm{keV}$ were obtained even under very intense 


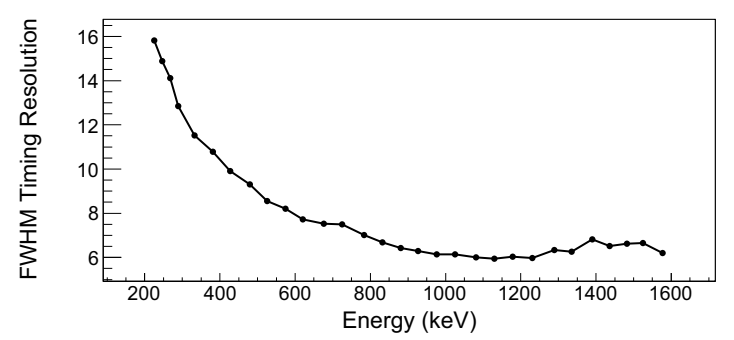

Figure 2. Shown in the upper panel is the $\mathrm{BaF}_{2}: \mathrm{HPGe}$ time resolution (FWHM) as a function of gamma-ray energy. This data was taken with cascades from capture and fission on ${ }^{239} \mathrm{Pu}$.

count rates. Further, time reconstruction of the events was sufficient for use with DANCE. Event times could be reconstructed within $8 \mathrm{~ns}$ for $50 \%$ of the HPGe data, $12 \mathrm{~ns}$ for $70 \%$ of the HPGe data, or $20 \mathrm{~ns}$ for $85 \%$ of the HPGe data. The achieved time resolution is shown as a function of gamma-ray energy in Figure 2. The timing reconstruction depends on correlations between correction factors in the pulse-shape defect. Higher energy signals could more reliably be reconstructed within a narrow time window. Details of this test and the analysis can be found in Ref. [16].

\section{Conclusions}

The DANCE data acquisition system is undergoing major upgrade. All of the present, 8-bit digitizers are being replaced with 14-bit digitizers with deeper on-board memory as well as on-board FPGAs. This upgrade is expected to expand the range of measurements that can be completed, improve the resolution and performance of the DANCE system, and simplify the coupling of DANCE to new auxiliary detectors, including PPAC fission tagging detectors and high-resolution gamma-ray detectors. It is planned that the upgrade will be complete in late 2015 .

\section{Acknowledgements}

We gratefully acknowledge the support of the U. S. Department of Energy through the LANL/LDRD Program and the Stockpile Stewardship Academic Alliance Program. This material benefited from the use of the Los Alamos Neutron Science Center and is based upon work supported by the U.S. Department of Energy, National Nuclear Security Administration under Contract Number DE-AC52-06NA25396.

\section{References}

[1] M. Heil, R. Reifarth, M.M. Fowler, R.C. Haight, F. Käppeler, R.S. Rundberg, E.H. Seabury, J.L. Ullmann, J.B. Wilhelmy, K. Wisshak, Nucl. Instr. and Meth. A 459, 229 (2001)

[2] M. Mocko, G. Muhrer, Nucl. Instr. and Meth. A 704, 27 (2013)
[3] P.W. Lisowski, C.D. Bowman, G.J. Russell, S.A. Wender, Nucl. Sci. Engineering 106, 208 (1990)

[4] E.I. Esch, R. Reifarth, E.M. Bond, T.A. Bredeweg, A. Couture, S.E. Glover, U. Greife, R.C. Haight, A.M. Hatarik, R. Hatarik et al., Phys. Rev. C 77, 034309 (2008)

[5] A.M. Alpizar-Vicente, T.A. Bredeweg, E.I. Esch, U. Greife, R.C. Haight, R. Hatarik, J.M. O’Donnell, R. Reifarth, R.S. Rundberg, J.L. Ullmann et al., Phys. Rev. C 77, 015806 (2008)

[6] M. Jandel, T.A. Bredeweg, E.M. Bond, M.B. Chadwick, A. Couture, J.M. O’Donnell, M. Fowler, R.C. Haight, T. Kawano, R. Reifarth et al., Phys. Rev. Lett. 109, 202506 (2012)

[7] S. Mosby, T.A. Bredeweg, A. Chyzh, A. Couture, R. Henderson, M. Jandel, E. Kwan, J.M. O’Donnell, J. Ullmann, C.Y. Wu, Phys. Rev. C 89, 034610 (2014)

[8] J.L. Ullmann, T. Kawano, T.A. Bredeweg, A. Couture, R.C. Haight, M. Jandel, J.M. O’Donnell, R.S. Rundberg, D.J. Vieira, J.B. Wilhelmy et al., Phys. Rev. C 89, 034603 (2014)

[9] J.L. Ullmann, E.M. Bond, T.A. Bredeweg, A. Couture, R.C. Haight, M. Jandel, T. Kawano, H.Y. Lee, J.M. O’Donnell, A.C. Hayes et al., Phys. Rev. C 87, 044607 (2013)

[10] J. Kroll, B. Baramsai, G.E. Mitchell, U. Agvaanluvsan, F. Bečváŕ, T.A. Bredeweg, A. Chyzh, A. Couture, D. Dashdorj, R.C. Haight et al., Phys. Rev. C 88, 034317 (2013)

[11] J.M. Wouters, A.A. Vicente, T.A. Bredeweg, E. Esch, R.C. Haight, R. Hatarik, J.M. O'Donnell, R. Reifarth, R.S. Rundberg, J.M. Schwantes et al., IEEE Transactions on Nuclear Science 53, 880 (2006)

[12] S. Ritt, P.A. Amaudruz, MIDAS - maximum integrated data acquisition system, unpublished, http://midas.psi.ch

[13] S. Mosby, F. Tovesson, A. Couture, D. Duke, V. Kleinrath, R. Meharchand, K. Meierbachtol, J. O’Donnell, B. Perdue, D. Richman et al., Nucl. Instr. and Meth. A 757, 75 (2014)

[14] C. Wu, A. Chyzh, E. Kwan, R. Henderson, J. Gostic, D. Carter, T. Bredeweg, A. Couture, M. Jandel, J. Ullmann, Nucl. Instr. and Meth. A 694, 78 (2012)

[15] Bredeweg, T. A., Agvaanluvsan, U., Becker, J. A., Bond, E. M., Couture, A. J., Ethvignot, T., Fitzpatrick, J. R., Fowler, M. M., Granier, T., Haight, R. C. et al., Recent actinide nuclear data efforts with the DANCE $4 \pi$ BaF2 array, in Proceedings of the International Conference on Nuclear Data for Science and Technology (2007), pp. 607-610, http: //dx . doi .org/10.1051/ndata: 07592

[16] K.T. Macon, A. Couture, S. Mosby, J.M. O’Donnell, J.C. Blackmon, Digital HPGe timing for DANCE, in preparation for Nucl. Instr. and Meth. A 
